\title{
Lacquer cracks observed in peripheral fundus of eyes with high myopia
}

This article was published in the following Dove Press journal:

International Medical Case Reports Journal

10 April 2017

Number of times this article has been viewed

\section{Mitsuki Suga \\ Kosei Shinohara \\ Kyoko Ohno-Matsui}

Department of Ophthalmology and Visual Science, Tokyo Medical and

Dental University, Tokyo, Japan
Correspondence: Kosei Shinohara Department of Ophthalmology and Visual Science, Tokyo Medical and Dental University, I-5-45 Yushima, Bunkyo-ku,

Tokyo II3-8510, Japan

$\mathrm{Tel}+8 \mid 358035302$

Fax +8I 338187188

Email shinohara.oph@tmd.ac.jp
Abstract: We report a case with lacquer cracks observed in the peripheral fundus. A 37-year-old patient with bilateral high myopia who visited our clinic was examined by fluorescein angiography (FA) and fundus autofluorescence (FAF) to determine whether there were myopic fundus lesions. FA showed many arch-shaped, hyperfluorescent linear lesions running circumferentially in the peripheral fundus. FAF showed hypo-autofluorescence at the corresponding sites. These characteristics were very similar to the lacquer cracks present in the posterior fundus in highly myopic eyes. Although lacquer cracks in the peripheral fundus are rare, they should be considered in the differential diagnosis of peripheral linear lesions showing hyperfluorescence in FA. Keywords: lacquer cracks, high myopia, peripheral fundus, fluorescein angiography, fundus autofluorescence

\section{Introduction}

Lacquer cracks are considered to be healed mechanical fissures in the retinal pigment epthelium (RPE)-Bruch's membrane-choriocapillaris complex and are seen ophthalmoscopically as yellowish linear lesions. ${ }^{1,2}$ We have reported the characteristics of lacquer cracks in the spectral-domain optical coherent tomographic (SD-OCT), fluorescein angiographic (FA), indocyanine green angiographic (ICGA), and fundus autofluorescence (FAF) images. ${ }^{3}$ All the findings were compatible with the suggested pathophysiology of healed mechanical fissures in the RPE-Bruch's membrane-choriocapillaris complex.

Lacquer cracks generally occur in the posterior pole of the fundus, and to date, there has been only one case report on lacquer cracks that appeared in the peripheral fundus. ${ }^{4}$ However, the description of these lacquer cracks was limited. We report a patient with high myopia with lacquer cracks observed in the peripheral fundus. We present the characteristics of the cracks observed in the FA and FAF images and also the three-dimensional magnetic resonance images (3D MRI) of both eyes of this patient.

\section{Case report}

A 37-year-old man visited the High Myopia Clinic of the Tokyo Medical and Dental University hospital with a complaint of decreased vision in both eyes. He had no prior medical or ophthalmological history and was not taking any medications. His visual acuity was 20/20 in both eyes, and the refractive error was -14.5 D OD (right eye) and -14.0 D OS (left eye). The axial length was $30.8 \mathrm{~mm}$ OD and $30.6 \mathrm{~mm}$ OS. Dilated fundus examination showed that the optic disk cups were enlarged in both eyes, but 
there were no abnormalities in the peripheral region ophthalmoscopically (Figure 1). He was suspected to have glaucoma in both eyes, and the Goldmann perimetry was performed. A slight reduction in the sensitivity in the nasal visual field of the left eye was found. FA and FAF were performed to determine whether the fundus was normal. FA showed multiple archshaped, hyperfluorescent linear lesions running circumferentially in the peripheral fundus of both eyes. Some of these lesions were observed to run parallel to each other (Figure 2). FAF showed hypoautofluorescence at the corresponding sites (Figure 3). From these findings, we diagnosed these lesions as lacquer cracks in the peripheral fundus. 3D MRI showed that the shapes of both eyes were normal without posterior staphylomas. However, the entire posterior region of the globe was enlarged in both eyes (Figure 4).

Written informed consent was obtained from the patient for publication of this report and any accompanying images. The local committee of Tokyo Medical and Dental University Hospital judged that the ethical review of this case report was unnecessary.

\section{Discussion}

We reported earlier that lacquer cracks appeared hyperfluorescent during the entire angiographic phase of FA because of the window defect in the early phase and tissue filling in the late phase. FAF did not show hypoautofluorescence due to the atrophied RPE. These findings corresponded with the pathophysiology of lacquer cracks that are considered to be healed mechanical fissures in the RPE-Bruch's membrane-

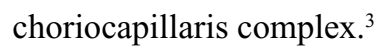

In the present case, FA showed hyperfluorescent, archshaped linear lesions running circumferentially in the peripheral fundus during the entire angiographic phase, and FAF showed hypoautofluorescence at the corresponding sites. These linear lesions need to be distinguished from angioid streaks, pigmented peripheral streaks, ${ }^{5}$ and choroidal rupture. Their ophthalmoscopic, FA, and FAF appearances were completely different from these lesions, and the patient had no history of trauma to both eyes. Although the direction and the area of the lesions were not typical of lacquer cracks, the FA and FAF findings were the same as those of

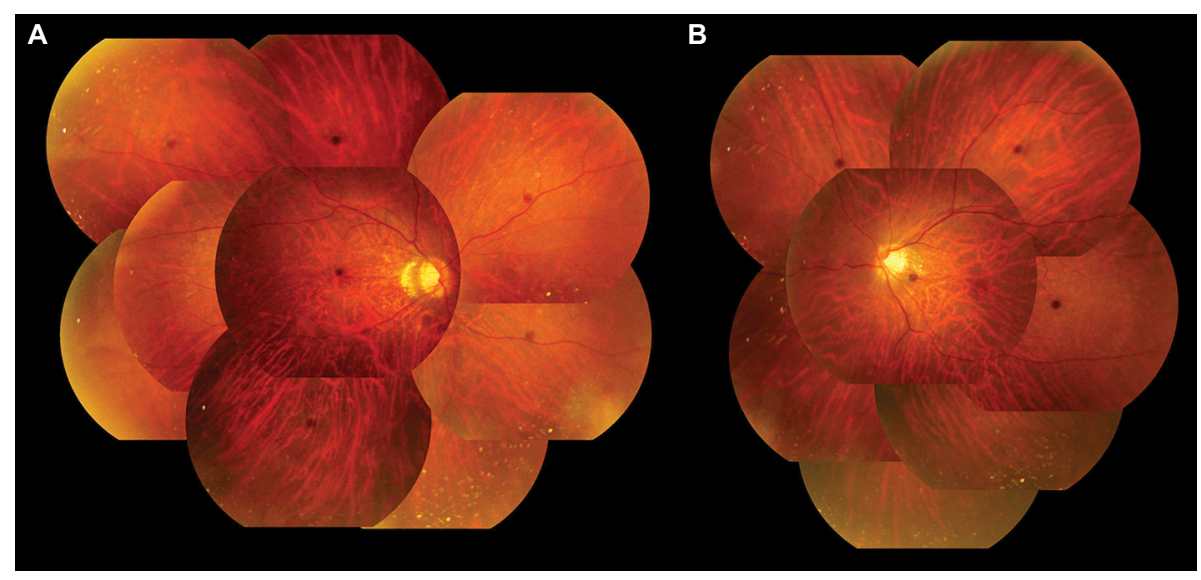

Figure I Color fundus photographs of the right $(\mathbf{A})$ and left $(\mathbf{B})$ eyes. Note: Both eyes do not show any obvious abnormalities in the peripheral region.

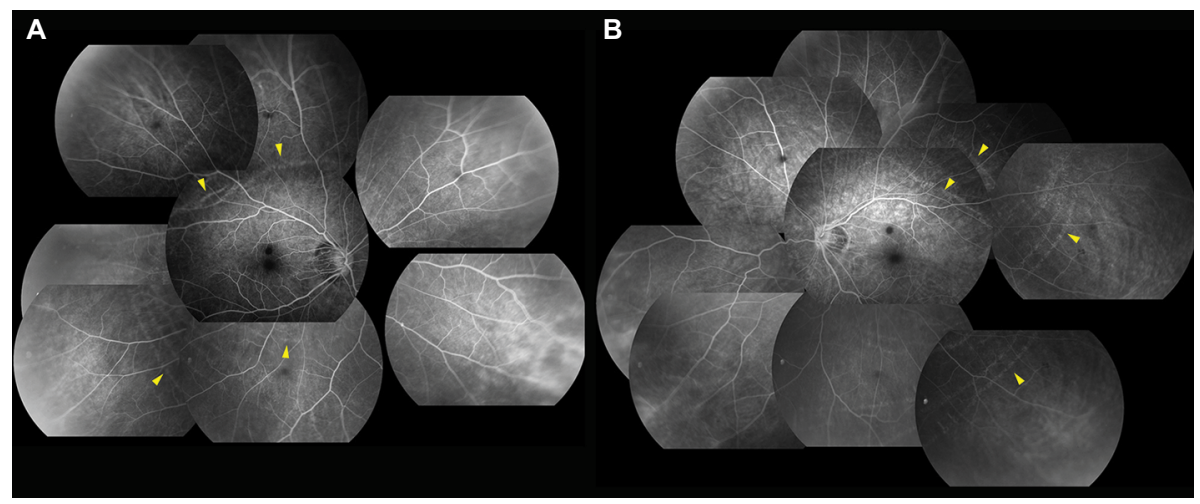

Figure 2 Fluorescein angiographic images of the right (A) and left (B) eyes.

Note: Many arch-shaped hyperfluorescent linear lesions running circumferentially are observed in the peripheral fundus of both eyes (arrowheads), and part of these lesions can be seen to run in parallel. 
A

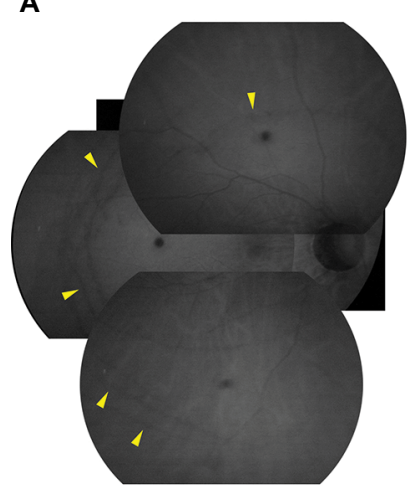

B

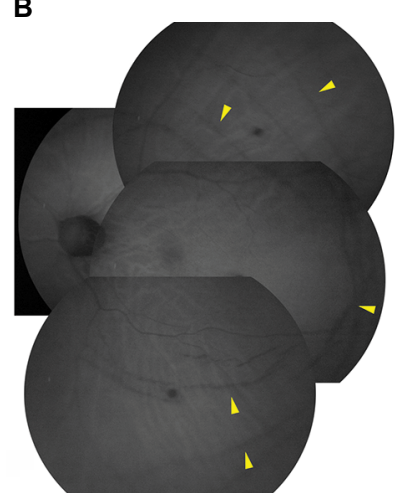

Figure 3 Fundus autofluorescence of the right (A) and left (B) eyes.

Note: Linear hypoautofluorescent lesions are observed at sites corresponding to the hyperfluorescence in the FA images (arrowheads).

Abbreviation: FA, fluorescein angiographic

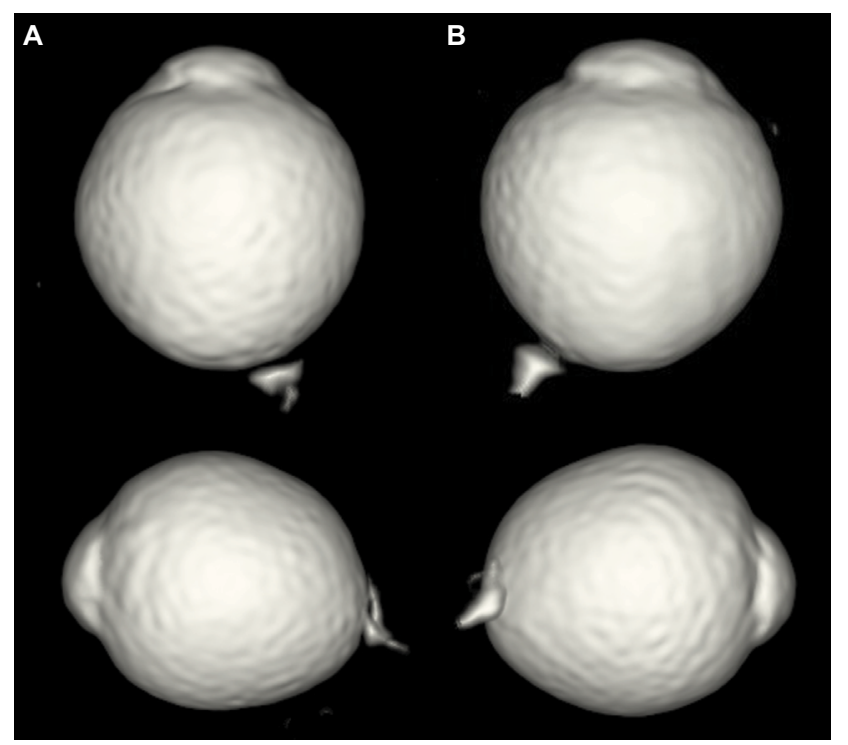

Figure 4 3D MRI images of the right (A) and left (B) eyes.

Notes: Top images, viewed from the inferior. Bottom images, viewed from the nasal side. The globes do not show any obvious protrusions, but the entire posterior regions are expanded.

Abbreviation: 3D MRI, three-dimensional magnetic resonance image.

lacquer cracks in the posterior fundus. Thus, these lesions were most likely also due to ruptures of the RPE-Bruch's membrane-choriocapillaris complex.

Lacquer cracks generally occur in the posterior pole of the fundus and are caused by an elongation of the globe. ${ }^{6}$ In the present case, 3D MRI did not show any protrusions of the globe indicating a posterior staphyloma, but instead the entire posterior globe was expanded. It might be possible that the expansion of the peripheral area was the cause of the lacquer cracks occurring in the peripheral fundus. With increasing time, lacquer cracks progress to other myopic

fundus changes including patchy atrophy, diffuse atrophy, and choroidal neovascularization. ${ }^{7}$ There have not been reports about peripheral myopic choroidal neovascularization before, and it is not certain whether peripheral lacquer cracks would cause myopic choroidal neovascularization in the future. However, we occasionally notice atrophic regions in relatively peripheral fundus in highly myopic eyes. These lesions might have resulted from peripheral lacquer cracks. Therefore, cases with peripheral lacquer cracks should be followed regularly with attention paid to whether the myopic fundus changes develop not only in the posterior pole but also in the peripheral region.

\section{Conclusion}

We report a case with peripheral linear lesions that have properties of the lacquer cracks found in the posterior pole of the eye. Although peripheral lacquer cracks are rare and are sometimes difficult to be detected by ophthalmoscopy, they should be considered in the differential diagnosis of peripheral linear lesions showing hyperfluorescence in FA.

\section{Acknowledgment}

The authors thank Professor Emeritus Duco Hamasaki at the Bascom Palmer Eye Institute, University of Miami, Florida, for his critical discussion and final manuscript revision.

\section{Disclosure}

The authors report no conflicts of interest in this work.

\section{References}

1. Grossniklaus HE, Green WR. Pathologic findings in pathologic myopia. Retina. 1992;12(2):127-133.

2. Klein RM, Green S. The development of lacquer cracks in pathologic myopia. Am J Ophthalmol. 1988;106(3):282-285.

3. Shinohara K, Moriyama M, Shimada N, Tanaka Y, Ohno-Matsui K. Myopic stretch lines: linear lesions in fundus of eyes with pathologic myopia that differ from lacquer cracks. Retina. 2014;34(3): 461-469.

4. Malagola R, Pecorella I, Teodori C, et al. Peripheral lacquer cracks as an early finding in pathological myopia. Arch Ophthalmol. 2006;124(12): 1783-1784.

5. Shinohara K, Moriyama M, Shimada N, Yoshida T, Ohno-Matsui K. Peripheral pigmented streaks in eyes with pathologic myopia. Retina. 2016;36(8):1573-1578.

6. Soubrane G, Coasas GJ. Choroidal neovascular membrane in degenerative myopia. In: Ryan SJ, editor. Retina. 4th ed. St. Louis, MO: Mosby; 2005:1136-1152.

7. Ohno-Matsui K, Ito M, Tokoro T. Subretinal bleeding without choroidal neovascularization in pathologic myopia. A sign of new lacquer crack formation. Retina. 1996;16(3):196-202. 
The International Medical Case Reports Journal is an international, peer-reviewed open-access journal publishing original case reports from all medical specialties. Previously unpublished medical posters are also accepted relating to any area of clinical or preclinical science. Submissions should not normally exceed 2,000 words or
4 published pages including figures, diagrams and references. The manuscript management system is completely online and includes a very quick and fair peer-review system, which is all easy to use. Visit http://www.dovepress.com/testimonials.php to read real quotes from published authors.

Submit your manuscript here: https://www.dovepress.com/international-medical-case-reports-journal-journal 